\title{
A Study on the Compensation of the Inductance Parameters of Interior Permanent-Magnet Synchronous Motors Affected by the Magnet Size
}

\author{
Ik-Sang Jang ${ }^{1}$, Hyung-Woo Lee ${ }^{2}$, Won-Ho Kim ${ }^{1}$, Su-Yeon Cho ${ }^{1}$, \\ Mi-Jung Kim ${ }^{1}$, Ki-Doek Lee ${ }^{1}$, and Ju Lee ${ }^{1 *}$ \\ ${ }^{1}$ Hanyang Univ., Haengdang-dong, Seongdong-gu, Seoul 133-791, Korea \\ ${ }^{2}$ Korea Railroad Research Institute, 360-1 Woram-dong, Uiwang-Si, Gyeongi-do 437-757, Korea
}

(Received 30 September 2010, Received in final form 10 March 2011, Accepted 10 March 2011)

\begin{abstract}
Interior permanent-magnet synchronous motors (IPMSMs) produce both magnetic and reluctance torques. The reluctance torque is due to the difference between the $d$ - and $q$-axis inductances based on the geometric rotor structure. The steady-state performance analysis and precise control of the IPMSMs greatly depend on the accurate determination of the parameters. The three essential parameters of the IPMSMs are the armature flux linkage of the permanent magnet, the d-axis inductance, and the q-axis inductance. In the basic design step of an IPMSM, the inductance parameters are very important for determining the motor characteristics, such as the input voltage, torque, and efficiency. Thus, it is very important to accurately estimate the values of the motor inductances. The inductance parameters of IPMSMs have nonlinear characteristics along the magnet size because the iron core is saturated by the magnet and armature reaction fluxes. In this study, the inductance parameters were calculated using both the magnetic-equivalent-circuit method and the finite-element method (FEM). Then the calculated parameters were compensated by the saturation coefficient function, which was also calculated via the magnetic-equivalent-circuit method and FEM.
\end{abstract}

Keywords : IPMSM, inductance parameters, PM motors, saturation coefficient

\section{Introduction}

Inductance parameters calculated via the magneticequivalent-circuit method were compared with those obtained from the finite-element method (FEM) with a different magnet size. When configuration of machine is complex and presence of saturation cannot be neglected, Magnetic equivalent circuit method is suffered. The dand q-axis inductances are calculated by permeance in the ideal magnetic circuit. The accuracy of this method depends greatly on the correct determination of saturation coefficient, which rely on the machine geometry [1]. Linear interpolation was used to compensate magnetic circuit method for the saturation coefficient.

\section{Inductance Calculation}

The magnet flux linkage is usually estimated from the back electromotive force (EMF). It is possible to estimate

*Corresponding author: Tel: +82-2-2220-0342

Fax: +82-2-2295-7111, e-mail: julee@hanyang.ac.kr accurate value of magnet flux rotor speed and induced voltage are measured precisely. However, estimation of daxis and q-axis inductances need more careful approach as it involves saturation and cross-coupling effects. There are various approaches to estimate d-axis and q-axis inductances such as magnetic equivalent circuit methods, finite-element analysis, and experimental measurements [2].

In magnetic equivalent circuit method, inductance parameters are a function of the magnetic width, thickness, and pole arc ratio. In the following equation, the saturation effect is neglected [3].

$$
L_{d}=\frac{3}{\pi} \frac{\mu_{0}\left(k_{\omega 1} N_{p h}\right)^{2} D l}{p^{2} g_{d}{ }^{\prime \prime}}, L_{q}=\frac{3}{\pi} \frac{\mu_{0}\left(k_{\omega 1} N_{p h}\right)^{2} D l}{p^{2} g_{q}{ }^{\prime \prime}},
$$

where $g_{d}{ }^{\prime \prime}$ : effective airgap length of d-axis and $g_{q}{ }^{\prime \prime}$ : effective airgap length of q-axis.

In FEM, the saturation can be studied appropriately, and the estimated inductances are fairly accurate. The d- and q-axis inductances can be obtained from the flux linkage vector diagram [4]. 


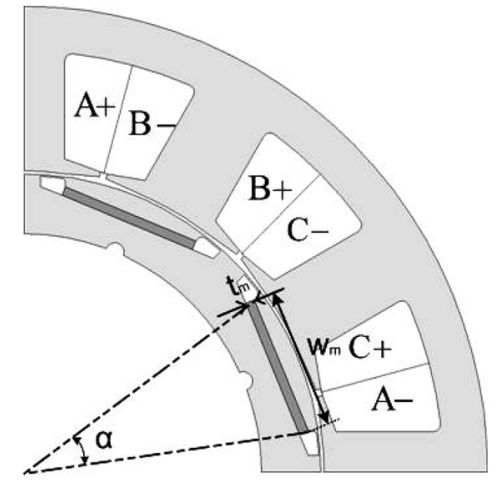

Fig. 1. Permanent-magnet variables.

$$
L_{d}=\frac{\Psi_{o} \cos \alpha-\Psi_{a}}{i_{d}}, L_{q}=\frac{\Psi_{o} \sin \alpha}{i_{q}}
$$

\section{Compensation of the Saturation Coefficients}

The inductances are affected by the magnet width and thickness. Fig. 1 shows these two variables.

The function of position $\left(x_{i}, y_{i}\right)$ is expressed as $f\left(x_{i}\right.$, $\left.y_{i}\right)=f_{i, j}$. This function is calculated from the values of points $\mathrm{A}$ and $\mathrm{B}$, as follows:

$$
\begin{aligned}
& f_{A}=f_{i, j}+s \Delta f_{i, j}=f_{i, j}+\frac{y-y_{i}}{k}\left(f_{i, j+1}-f_{i, j}\right), \\
& f_{B}=f_{i+1, j}+\frac{y-y_{i}}{k}\left(f_{i+1, j+1}-f_{i+1, j}\right), \text { and } \\
& f_{x, y}=f_{A}+s \Delta f_{A}=f_{A}+\frac{x-x_{i}}{h}\left(f_{B}-f_{A}\right),
\end{aligned}
$$

where $h$ : distance between $x_{i}$ and $x_{i+1}$ and $k$ : distance between $y_{i}$ and $y_{i+1}$.

Saturation coefficient $k_{\text {sat }}$ is defined from FEM and the magnetic-equivalent-circuit method as follows:

$$
k_{\text {sat }}=\frac{L_{d}(\mathrm{FEM})}{L_{d}(\mathrm{Mag})} .
$$

Table 1 shows the initial four points that are the base points for interpolation.

From these points, the following saturation coefficient functions are obtained:

$$
\begin{aligned}
& k_{\text {sat }}^{S 60}(x, y)=-175.82 x y+66.91 x-1.21 y+1.03 \\
& k_{\text {sat }}^{\text {S08 }}(x, y)=-269.95 x y+105.75 x-y+0.97,
\end{aligned}
$$

where $x=t_{m}^{n}$ and $y=w_{m}^{n}$.

Fig. 2(a) and (b) show the saturation coefficients for the
Table 1. Initial four points for interpolation.

\begin{tabular}{ccclc}
\hline \hline$w_{n m}$ & $t_{n m}$ & $\begin{array}{c}L_{d} \\
\text { (Magnetic Circuit) }\end{array}$ & $\begin{array}{c}L_{d} \\
(\mathrm{FEM})\end{array}$ & $k_{\text {sat }}$ \\
\hline \multicolumn{5}{c}{$\mathrm{S} 60$} \\
0.26 & 0.01 & 0.3850 & 0.3591 & 0.9326 \\
0.26 & 0.03 & 0.1817 & 0.2465 & 1.3565 \\
0.30 & 0.01 & 0.4524 & 0.36824 & 0.8139 \\
0.30 & 0.03 & 0.2250 & 0.2469 & 1.0972 \\
\hline & \multicolumn{5}{c}{$\mathrm{S} 08$} & 0.4092 & 1.1311 \\
\hline 0.26 & 0.01 & 0.3850 & 0.3223 & 1.7738 \\
0.26 & 0.03 & 0.1817 & 0.4138 & 0.9145 \\
0.30 & 0.01 & 0.4524 & 0.3172 & 1.4098 \\
0.30 & 0.03 & 0.2250 &
\end{tabular}

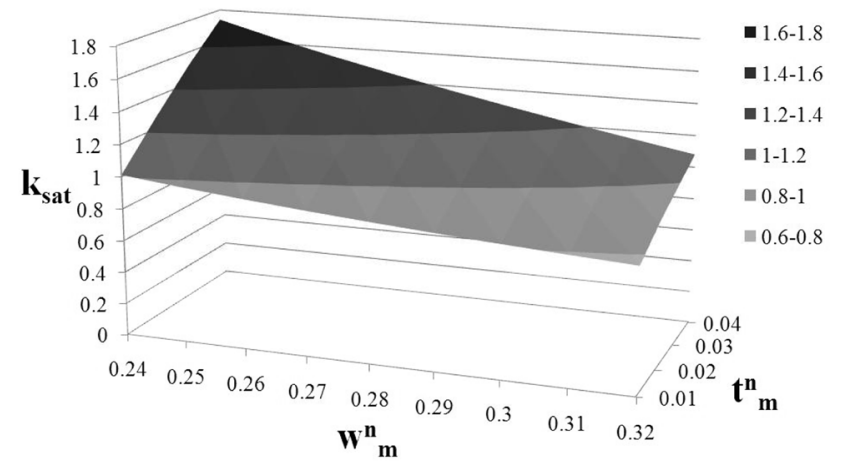

(a)

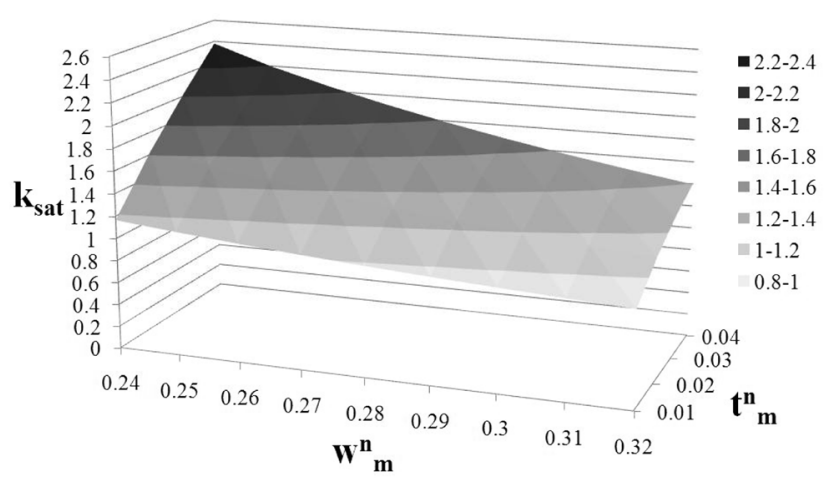

(b)

Fig. 2. Comparison of the saturation coefficients: (a) S60 core; (b) S08 core.

S60 core (saturation level: 1.7 Tesla) and the S08 core (saturation level: 1.6 Tesla), respectively.

\section{Conclusion}

In this study, the inductance parameters calculated via the magnetic-equivalent-circuit method were compensated 
using the saturation coefficient. This coefficient was obtained via linear interpolation. Only four executions of FEM analysis are required to obtain an interpolated coefficient function. Thus, this method is useful for determining the magnet size in the basic design step.

\section{Acknowledgement}

This work was supported by the National Research Foundation of Korea (NRF) grant funded by the Korea government (MEST) (No. 2008-0060145).

\section{References}

[1] J. F. Gieras, E. Santini, and M. Wing, IEEE Trans. Magn. 34, 3712 (1998).

[2] Rukmi Dutta and M. F. Rahman, IEEE Trans. Magn. 42 (2006).

[3] Jung Ho Lee, Jung Chul Kim, and Dong Seek Hyun, IEEE Trans. Magn. 35, 1199 (1999).

[4] Chang-Sung Jin, Dae-Sung Jung, Ki-Chan Kim, Yon-Do Chun, Hyung-Woo Lee, and Ju Lee, IEEE Trans. Magn. 45, 2811 (2009). 